\title{
UV-triggered photoinsertion of contrast agent onto polymer surfaces for in vivo MRI-visible medical devices
}

Anita Schulz ${ }^{1}$, Laurent Lemaire ${ }^{2}$, Audrey Bethry ${ }^{1}$, Lucie Allègre ${ }^{1,3}$, Maïda Cardoso ${ }^{4}$, Florence Bernex $^{5}$, Florence Franconi ${ }^{2}$, Christophe Goze-Bac ${ }^{4}$, Hubert Taillades ${ }^{6}$, Xavier Garric ${ }^{1}$, Benjamin Nottelet ${ }^{1 *}$

${ }^{1}$ IBMM, Univ Montpellier, CNRS, ENSCM, Montpellier, France.

${ }^{2}$ Micro \& Nanomédecines Translationnelles-MINT, Univ Angers, INSERM U1066, CNRS UMR 6021, Angers, France ; PRISM Plate-forme de recherche en imagerie et spectroscopie multi-modales, Angers, France

${ }^{3}$ Service de Gynécologie Obstétrique, CHU de Nîmes, Univ Montpellier, Nîmes, France

${ }^{4}$ L2C, BioNanoImaging Foundry, Univ Montpellier, CNRS, ENSCM, Montpellier, France.

${ }^{5}$ [RHEM], BioCampus Montpellier, CNRS, INSERM, Univ Montpellier, Montpellier, France and IRCM, U1194 INSERM, Univ Montpellier, Montpellier, France

${ }^{6}$ Plateau de simulation, UFR Medecine, Univ Montpellier, Montpellier, France

E-mail: Benjamin.nottelet@umontpellier.fr 


\begin{abstract}
Polymeric materials are largely employed for the manufacturing of implants for various reasons, but they are typically invisible by conventional imaging methods. To improve surgical procedure and postoperative implant follow-up though, biomaterials are needed which allow an accurate and efficient imaging. Here, we present a direct and versatile strategy that allows to covalently immobilize T1 magnetic resonance imaging (MRI) contrast agents at the surface of various clinically relevant polymeric biomaterials. An aryl-azide bearing complex of 1,4,7,10tetraazacyclododecane-1,4,7,10-tetraacetic acid (DOTA) and gadolinium (Gd) has been synthesized for easy photografting onto polymer surfaces. Polycaprolactone (PCL), polylactide (PLA), polyurethane (PU), polyetheretherketone (PEEK) and polypropylene (PP) have been selected as clinically relevant substrates and successfully functionalized with the photosensitive MRI probe DOTA/Gd. Following in vitro assessment of their biocompatibility and MRI visibility, commercial MRI-visible PP hernia repair meshes (MRI-meshes) have been prepared. MRI-meshes have been implanted in rats for in vivo evaluation of their imaging capacities over 1 month. Histological evaluation and Gd biodistribution studies have been carried out confirming the potential of this straightforward approach to simply yield imageable medical devices.
\end{abstract}

Keywords: in vivo medical resonance imaging (MRI), polymer surface, medical device, surgical mesh, photomodification, gadolinium-DOTA 


\section{Introduction}

Implantable medical devices (IMD) represent a keystone of modern medicine and will continue to be essential to human health improvement as demonstrated by the increasing demand (eg. among elderly population) that supports a $5 \%$ annual growth. ${ }^{1-2}$ Among them, polymeric IMDs represent one of the major class along with metallic or ceramic ones. This preponderance is explained by the fact that polymeric biomaterials can be tailored to most requirements of the target medical applications: durability or degradability, mechanical properties ranging from rigid to soft, ease of process and sterilization and so forth. Polymeric IMDs are therefore present in all surgical specialties including orthopedics, cardiovascular, ocular, visceral or gynecological surgeries. One main drawback remains however the quasi-absence of medical imaging possibilities of the polymeric IMDs. This is a strong limitation considering that imageguided surgical procedures are increasingly used for improved outcomes of IMDs implantation. $^{3-7}$ Making polymeric IMDs visible under clinical imaging modalities can therefore help to increase their implantation success rates, but also allow accurate and efficient postoperative imaging to evaluate migration, shrinkage, or even fate in the case of temporary IMDs. ${ }^{8-11}$ It is therefore of prime importance to propose biomaterials designed to meet the criteria of current clinical imaging, subsequently reducing the incidence of complications and facilitating early diagnostics.

All imaging modalities are potentially affected by such developments, ${ }^{12}$ however magnetic resonance imaging (MRI), and X-ray radiography/computed tomography (CT) remain to date the predominant fields of investigations when clinical outcomes are foreseen. Despite their low sensitivity compared to other imaging modalities, they offer a combination of convenient noninvasive application, high spatial resolution, absence of limit on tissue penetration depth, tomographic capability and relative low cost. As a consequence, X-ray and MRI contrast agents (CA) of various natures have been developed in the past and are still used in the clinic such as metal rings for enhanced angioplasty procedures, sulfate barium loaded thermoplastics to produce radiopaque IMDs and surgical tools or the routine gadolinium-based blood pool contrast agents. However, these CAs exhibit inherent drawbacks either in terms of spatial resolution (e.g. metallic rings that do not allow visualization of the whole IMD), temporal resolution (leaching of molecular CAs), or toxicity (e.g. acute renal toxicity or adverse allergic and pseudo-allergic reactions for X-ray CAs). ${ }^{13-14}$ In particular, the use of MRI CAs based on gadolinium $(\mathrm{Gd})$ is in debate. The main reason being at risk of nephrogenic systemic fibrosis (NSF), which is associated with the toxicity of free $\mathrm{Gd}(\mathrm{III})$. Despite a low occurrence that is 
limited to renal impaired patients and mostly to the first generation of linear chelating agents, NFS has been the first threat upon Gd-based CAs. ${ }^{15}$ In addition, recent reports mentioning Gd accumulation in the brain may further sound the death knell for Gd-based CAs ${ }^{16}$ as confirmed by the recent restrictions and even suppression of some linear gadolinium agents following the conclusions of the European Medicines Agency. ${ }^{17}$ It is therefore mandatory to propose alternatives to low molecular weight Gd-based CAs for MRI medical imaging of IMDs or find methods allowing imaging with limited doses of CAs.

For instance, one approach relies on the development of specific MRI-sequences that can allow for the detection of biomaterials without addition of CAs. Commercial polyethylene terephthalate meshes coated with collagen have for example been visualized in vivo in preclinical models using amide-proton transfer MRI. ${ }^{18}$ Although interesting and promising, this approach is still limited to research and will require significant technological developments of imaging instruments before translation into clinic. Replacement of Gd-complexes by other and safer metal-complexes, for example Fe-complexes as T1 contrast agents, is also currently under investigation $^{19}$. Another strategy is the development of MRI-visible polymers used as macromolecular contrast agents that can be embedded in the IMD or used as coatings. This includes the use of commercially available polymers comprising fluorine (e.g. polyvinylidene fluoride, PVDF) for ${ }^{19} \mathrm{~F} \mathrm{MRI}^{20-21}$ or synthesis of new copolymers integrating an MRI probe in the polymer chain like fluorinated repeating units, ${ }^{22-25}$ or nitroxide groups. ${ }^{26}$ However, again, fthe translation into the clinic requires the development of dedicated clinical tools as well as approval of these new contrast agents. In an attempt to circumvent these drawbacks, various macromolecular contrast agents embedding the existing and approved contrast agents (DTPA or DOTA) as repeating units or chain-ends have been designed. ${ }^{12}$ In this context, hydrophobic coatings for IMDs based on degradable poly $(\varepsilon \text {-caprolactone })^{27-28}$ or biostable poly(methyl (meth)acrylate) $)^{29-30}$ have been developed. This approach has the advantage of ensuring long term visibility of the IMDs while drastically decreasing the required amount of Gd, which guaranties low if any toxicity compared to repeated bolus injection of Gd-based CAs. ${ }^{31}$ However, it requires an additional step of coating which can potentially be associated to delamination depending on the nature of the IMD.

An elegant and effective alternative would consist in the surface modification of IMDs to immobilize CAs at their surface. Yet, to the best of our knowledge, no simple surface modification methodology exists to date that is applicable to a large variety of polymeric biomaterials and that allows their functionalization to make them MRI-visible while 
maintaining their intrinsic functionalities and properties. We recently reported on the use of photo-reactive aryl-azide moieties and their potential to easily impart surface properties to a large variety of polymeric substrates. ${ }^{32}$ Taking advantage of this methodology, we present here a direct and versatile strategy that allows to covalently immobilize T1 MRI CAs at the surface of various clinically relevant biomaterials. In more detail, the synthesis of an aryl-azide bearing complex of 1,4,7,10-tetraazacyclododecane-1,4,7,10-tetraacetic acid (DOTA) and Gd (MRIclip) and its photografting are described. Following an initial in vitro assessment of this approach in terms of biocompatibility and MRI visibility, its potential is demonstrated in vivo with modified commercial polypropylene hernia repair meshes (MRI-meshes). Visualization of mesh over 1 month, histological and Gd biodistribution evaluations are used to confirm the potential of this straightforward approach to yield imageable medical devices.

\section{Methods}

\subsection{Materials and methods}

4-azidoaniline hydrochloride was purchased from TCI Europe (Zwijndrecht, Belgium) and 2,2',2"-(10-(2,6-dioxotetrahydro-2H-pyran-3-yl)-1,4,7,10-tetraazacyclododecane-1,4,7triyl)triacetic acid (DOTA-GA anhydride) was purchased from Chematech (Dijon, France). All other materials were obtained from Sigma Aldrich (St Quentin Fallavier, France) and were used without further purification. Medical grade polypropylene (PP) used for surgical mesh for hernia repair was obtained from Luxilon (Antwerp, Belgium). Medical grade poly(ether ether ketone) (PEEK-Optima, Invibio) films were kindly provided by AO Research Institute. Poly(lactide) (PLA) was synthesized by bulk-ring opening copolymerization of L-lactide (92\%) and DL-lactide (8\%) (PURAC, Lyon, France) using tin 2-ethylhexanoate as catalyst. PLA and PP were pressed into $0.5-2 \mathrm{~mm}$ thick disks at $180^{\circ} \mathrm{C}$ and $5 \mathrm{t}$ for $30-45 \mathrm{~min}$ using a Carver Manual Bench Top Laboratory Press and subsequently cut into disks or films. Polyurethane (PU) films were cut from a percutaneous nephrostomy catheter (Rüsch, 340014), PP meshes samples were cut out Dynamesh-PP meshes.

ICP-MS Analyses. $\mathrm{Gd}^{3+}$ was quantified using an Element XR sector field ICP-MS (inductively coupled plasma-mass spectrometry) at Géosciences in Montpellier (University of Montpellier). Internal standardization used an ultrapure solution enriched with indium.

${ }^{1} \mathrm{H}$ and ${ }^{13} \mathrm{C}$ NMR analyses. NMR analyses were carried out on a BRUKER Avance III - 500 $\mathrm{MHz}$ spectrometer. 
2.2. Synthesis of photoreactive 2-(4,7,10-triacetic acid)-1,4,7,10-tetraazacyclododecan-1yl)pentanedioic acid gadolinium complex (Gd-DOTA- $\left.N_{3}\right)$

Functionalization of DOTA was performed as described elsewhere. ${ }^{33}$ In detail, in an evacuated schlenk-flask $42.0 \mathrm{mg}$ (0.246 mmol, 1.2 eq.) 4-azidoaniline hydrochloride was dissolved in $2 \mathrm{~mL}$ anhydrous DMF. $34.5 \mu \mathrm{L}$ (0.249 mmol, 1.2 eq.) TEA and $100 \mathrm{mg}$ (0.202 mmol, 1 eq.) DOTA-GA anhydride (2,2',2"-(10-(2,6-dioxotetrahydro-2H-pyran-3-yl)-1,4,7,10tetraazacyclododecane-1,4,7-triyl)triacetic acid) were added. The reaction mixture was stirred for $2 \mathrm{~h}$ at $45^{\circ} \mathrm{C}$ and continued to be stirred at room temperature (RT) overnight. The solvent was removed under high vacuum and mild heating. The residue was dissolved in $2 \mathrm{~mL}$ $\mathrm{MeOH} / \mathrm{CHCl}_{3}(2 / 1 ; \mathrm{v} / \mathrm{v})$ and precipitated with $2 \mathrm{~mL}$ diethyl ether. Subsequently the precipitate was centrifuged, dried, dissolved in $\mathrm{H}_{2} \mathrm{O}$ and lyophilized to obtain the product as a yellowbrownish powder $(85 \%)$.

${ }^{1} \mathrm{H}$ NMR (500 MHz, MeOD, $\delta$ ): 7.57 (d, -CH-); 7.33 (m, -CO-NH-); 7.01 (d, -CH-); 3.95-2.69 (m, -CO-CH${ }_{2}-\mathrm{N}-;-\mathrm{CH}_{2}-\mathrm{N}-;-\mathrm{CH}_{2}-\mathrm{CH}-\mathrm{N}-;-\mathrm{CO}_{-} \mathrm{CH}_{2}-\mathrm{CH}_{2}$ ); 2.15-1.95 (m, - $\left.\mathrm{CH}_{2}-\mathrm{CH}-\mathrm{N}-;\right)$ (Figure S1). ${ }^{13} \mathrm{C}$ NMR (500 MHz, MeOD, $)$ ): 179 and $177(-\mathrm{CHCOOH}$, isomer 1 and 2, respectively); 174 and 173 (-CONH-, isomer 1 and 2, respectively); 171 (- $\left.\mathrm{CH}_{2} \mathrm{COOH}\right) ; 143$ $\left(\mathrm{CH}-\mathrm{N}_{3}\right) ; 130$ (aromatic $\mathrm{CH}-\mathrm{NHCO}$ ); 123 and 120 (aromatic $\mathrm{CH}$ ); 64 and $61(-\mathrm{CHCOOH}$ isomer 1 and 2, respectively); $57\left(-\mathrm{CH}_{2} \mathrm{COOH}\right) ; 56\left(\mathrm{CH}_{2}-\mathrm{N}(\mathrm{CH})-\mathrm{CH}_{2}\right) ; 54$ and $51\left(\mathrm{CH}_{2}-\right.$ $\left.\mathrm{N}\left(\mathrm{CH}_{2}\right)-\mathrm{CH}_{2}\right) ; 33$ and $32\left(\mathrm{CH}_{2}-\mathrm{CH}_{2}-\mathrm{CONH}\right) ; 21.5$ and $20.5\left(\mathrm{CH}_{2}-\mathrm{CH}_{2}-\mathrm{CONH}\right)$ (Figure $\left.\mathrm{S} 2\right)$. TOF MS ES+ m/z: 593.27 calculated for $\left[\mathrm{C}_{25} \mathrm{H}_{37} \mathrm{~N}_{8} \mathrm{O}_{9}\right]^{+}$found at 593.27 and its fragments at $565.26\left[\mathrm{C}_{25} \mathrm{H}_{37} \mathrm{~N}_{6} \mathrm{O}_{9}\right]^{+}, 347.19\left[\mathrm{C}_{14} \mathrm{H}_{27} \mathrm{~N}_{4} \mathrm{O}_{6}\right]^{+}, 283.14\left[\mathrm{C}_{25} \mathrm{H}_{38} \mathrm{~N}_{6} \mathrm{O}_{9}\right]^{2+}, 219.08\left[\mathrm{C}_{11} \mathrm{H}_{15} \mathrm{~N}_{4} \mathrm{O}\right]^{+}$.

The complexation of DOTA-N 3 was done under various conditions (Table S1). In general, DOTA- $\mathrm{N}_{3}$ and $\mathrm{GdCl}_{3} \cdot 6 \mathrm{H}_{2} \mathrm{O}$ were dissolved in $\mathrm{H}_{2} \mathrm{O}$, which was subsequently adjusted to $\mathrm{pH} 5$ 7 with $1 \mathrm{M} \mathrm{NaOH}$. In a typical procedure (Table S1, last row), $34.8 \mathrm{mg}$ of DOTA-N 3 and 20.6 $\mathrm{mg}$ of $\mathrm{GdCl}_{3} \cdot 6 \mathrm{H}_{2} \mathrm{O}$ were used and the final solution volume was of $5 \mathrm{~mL}$. The reaction mixture was shaken for $2 \mathrm{~h}$ to $16 \mathrm{~h}$ at various temperatures (RT to $80^{\circ} \mathrm{C}$ ). The solution was diluted with more $\mathrm{H}_{2} \mathrm{O}$ and treated with Chelex 100 to remove free $\mathrm{Gd}$. The treatment was repeated until free $\mathrm{Gd}$ was below $15 \mu \mathrm{M}$ in a $1 \mathrm{~g} / \mathrm{L}$ solution of Gd-DOTA- $\mathrm{N}_{3}$ as detected with the methyl thymol blue (MTB) test. ${ }^{34} \mathrm{Gd}$ content ranged from 14-30\%. 


\subsection{Preparation of MRI-visible polymer surfaces by photomodification}

Clean polymer (e.g. PLA, PLA-Pluronic-PLA, PLGA, PCL, PP, PEEK, PU) surfaces (film, mesh, pellet) heated to temperatures from RT to $80^{\circ} \mathrm{C}$ were spray coated with $5 \mathrm{~g} / \mathrm{L}$ Gd-DOTA$\mathrm{N}_{3}$ dissolved in degassed $\mathrm{MeOH}$, air dried and irradiated for 20 min using a chromatography lamp VL-4C (254 nm, 8W) which was positioned 2-3 cm above the surface. Subsequently the surface was rinsed with $\mathrm{H}_{2} \mathrm{O}$ and $\mathrm{EtOH}$. Based on our previous work ${ }^{32}$ the modification step was repeated 5 times to increase surface coverage. Final purification was realized by ultrasonication in deionized water for $20 \mathrm{~min}$.

\subsection{Evaluation of biocompatibility of the polymer surfaces modified by Gd-DOTA-N}

Fibroblasts L929 cells (ECACC 85011425) were maintained in DMEM high glucose supplemented with 5\% Fetal Bovine Serum (FBS), 2mM L-glutamine and $1 \%$ penicillin/streptomycin and cultured at $37^{\circ} \mathrm{C}$ and $5 \% \mathrm{CO}_{2}$. Cells were tested mycoplasms-free.

\subsubsection{Cytotoxicity assay via direct contact method}

L929 cells were seeded at $1.7 \times 10^{4}$ cells per well in a 24-well plate and allowed to attach overnight under appropriate atmosphere. Polymers PLA and PP with and without Gd complex were cut in order to cover about $1 / 10$ of the well surface (as mentioned in ISO 10993-5 guidelines). Decontamination was realized: first step with ethanol $70 \%$ followed by 3 washing steps with PBS-penicillin/streptomycin 10\% and then PBS only.

The cell growth medium was replaced and the decontaminated polymer films were placed on top. After $48 \mathrm{~h}$ incubation under appropriate atmosphere, polymers were removed and cell viability was assessed by Prestoblue ${ }^{\circledR}$ cell viability assay (Invitrogen, A13261) according to manufacturer's instructions. Briefly, Prestoblue ${ }^{\circledR}$ was added at $10 \%$ in growth medium and after 45 minutes incubation fluorescence at 590nm was measured using CLARIOstar® (BMG LABTECH's) microplate reader. Wells without addition of polymers films were used as controls. $(n=4)$

\subsubsection{Cell proliferation assay}

Polymers PLA and PP with and without Gd complex were cut in order to cover 24-well plate surfaces $\left(1.9 \mathrm{~cm}^{2}\right)$. Polymer discs were swabbed with paper soaked with ethanol $70 \%$ and then rinsed with 3 baths of PBS-penicillin/streptomycin 10\% and then with PBS only. 
L929 cells were seeded on the surface of polymer held by o-ring, at $2 \times 10^{5}$ cells per well (drop of $40 \mu \mathrm{L}$ in the center of the well) in a 24 -well non-treated plate and incubated under appropriate atmosphere for about $2 \mathrm{~h}$, time for cells to adhere. Then after rinsing off non-adherent cells with PBS, fresh growth medium was added. Cell proliferation at 24, 48 and $120 \mathrm{~h}$ was assessed by using Prestoblue ${ }^{\circledR}$ cell viability assay (Invitrogen, A13261) according to manufacturer's instructions. Tissue culture polystyrene wells treated or not treated for cell proliferation (TC treated and TC non treated) without addition of polymers films were used as controls $(n=4)$.

\subsection{In vitro MRI imaging}

In vitro MRI evaluation of MRI-visible polymer plates was performed using a 7T BIOSPEC 70/20 Bruker machine equipped with BGA12 gradients $(675 \mathrm{mT} / \mathrm{m})$ and a bird-cage type 35$\mathrm{mm}$ resonator. In the case of MRI-visible PP meshes, they were also characterized on a $9.4 \mathrm{~T}$ Agilent DD2 system operating at a frequency of $400 \mathrm{MHz}$ with GC156/100 gradients (400 mT/m) and a Quadrature Volume Coil with an internal diameter of $43 \mathrm{~mm}$ from Rapid Biomedical. Samples were embedded in a degassed $1 \%(\mathrm{w} / \mathrm{w})$ low-gelling point agarose gel prior to imaging. Figure 3 presents the images recorded on the 7T Bruker MRI scanner using a 3 D-spin echo sequence (Field Of View FOV $3 \times 3 \times 1 \mathrm{~cm}$ matrix $128 \times 128 \times 48$, TR $=3000$ $\mathrm{ms}, \mathrm{TE}=8 \mathrm{~ms}(\mathrm{TEeff}=16 \mathrm{~ms})), \mathrm{RF}=8$, total acquisition time of 0:51) with an inversion delay of $1300 \mathrm{~ms}$ to allow for the nulling of the gel as previously described. ${ }^{27}$ Figure 4 presents the images recorded on the 9.4 $\mathrm{T}$ Agilent MRI scanner using a multiple spin echo multi slices protocol with an inversion delay $(\mathrm{TI}=985 \mathrm{~ms})$, a repetition time $(\mathrm{TR}=3000 \mathrm{~ms})$, an echo time $(\mathrm{TE}=10,21 \mathrm{~ms})$, a FOV of $40 \mathrm{~mm} \times 40 \mathrm{~mm}$ with a thickness of $0.5 \mathrm{~mm}$ (no gap) and an acquisition matrix $\left(\mathrm{N}_{\mathrm{READ}} \times \mathrm{N}_{\mathrm{PHASE}}\right)=192 \times 192$. All MRI processing and visualization were done using homebuilt programs under Matlab environment and ImageJ. This protocol was repeated on the 9.4T MRI Agilent scanner after a storage of 6 and 12 -months at $4{ }^{\circ} \mathrm{C}$, in order to study the stability of the implant with time.

\subsection{In vivo experiments}

\subsubsection{Animals}

All experiments were conducted in the experimental laboratory of the Faculty of Medicine of University of Montpellier. Seven Wistar Han female rats, weighing 200-220g, were purchased from the Charles River Laboratories ${ }^{\circledR}$ (L’Arbresle, France). Wistar Han rats were chosen 
because of their robustness and their low cost compared to large animal. Furthermore, their corpulence was most adapted to MRI available in our center.

All investigations were carried out in strict accordance with the recommendations in the Guide for the Care and Use of Laboratory Animals. The protocol was approved by the Committee on the Ethics of the French Ministry of Education and Research (approval $n^{\circ} 201605111534248$ ). All efforts were made to minimize animal suffering and to use the minimum number of animals necessary to produce reliable scientific data. All the animals were in quarantine for one week prior to treatment. They were housed in individual cages in a room at $22^{\circ} \mathrm{C}$ with a humidity rate of $55 \%(+/-10 \%)$ with free access to food (SAFE®) and tap water. They were examined, weighed and their litter changed daily, respecting the guide of good practices and animal welfare.

\subsubsection{Surgical procedure for mesh implantation}

Four female Wistar rats (Charles River Laboratories) were used for mesh implantation (Group 1). The rats were anesthetized by an intra-peritoneal injection of ketamine $(80 \mathrm{mg} / \mathrm{kg})$ and xylazine $(5 \mathrm{mg} / \mathrm{kg})$. A second injection of ketamine was performed if necessary. Animals were placed in a ventral position, the back area was shaved and prepared with iodine solution and then they were draped in a sterile fashion. A subcutaneous injection of xylocaine $(0.1 \%)$ was made to minimize post-operative pain. A cutaneous incision of $2 \mathrm{~cm}$ was made on each side of the backbone $1 \mathrm{~cm}$ below the chest. The fascia of lumbar muscle was incised in both sides and an interspace was created in the muscles by dissection.

We inserted a $1 \mathrm{~cm} * 1,5 \mathrm{~cm}$ polypropylene MRI-visible mesh in the right interspace, a $1 \mathrm{~cm} *$ $1,5 \mathrm{~cm}$ polypropylene control mesh was inserted in the left area. The muscle layer was closed by continuous suture of $3 / 0$ absorbable suture and the skin was closed by continuous suture of 3/0 absorbable suture.

Three female Wistar rats (Charles River Laboratories) were used as controls (Control Group). They underwent surgical procedure without any mesh implantation, two incisions were made as previously described and then closed with absorbable suture.

To avoid any bias in surgical procedure especially in implantation sites, the same surgeon implanted all the meshes. 
Post-operative monitoring was performed daily including assessment of the overall condition, of the weight and of the skin healing. If there were signs that could indicate a physical suffering of the animal (apathy, prostration, weight loss $>15 \%$, evisceration), euthanasia would have been performed. MRI images were performed at one week, two and four weeks under isoflurane anesthesia.

\subsubsection{Explantation, histological evaluation and biodistribution}

Rats of Group 1 were euthanized after a 1-month postoperative period by an intra-peritoneal injection of pentobarbital $(0.5 \mathrm{~mL} / \mathrm{kg})$. All meshes were explanted with surrounding tissues. Samples were immersed in $10 \%$ formalin at RT for 24 hours, washed twice in Phosphatebuffered saline solution and then immersed in $70 \%$ ethanol and kept at $4^{\circ} \mathrm{C}$. Then tissue samples were embedded in paraffin. Paraffin blocks were cut into $3 \mu \mathrm{m}$ thick sections. Prepared sections were then stained with hematoxylin-eosin-safran HES ((RHEM histology facility, Montpellier). Lesion intensity (i) and spreading (d) were graded by one senior pathologist (FB), blinded to the origin of the samples. Lesion intensity was graded from i0 (absence) to i4 (severe) according to the presence of inflammatory zones. Lesion spreading was evaluated from d0 (no lesion) to d2 (peripheral distribution). Results were compared with the control rats.

To detect Gd release from the MRI-visible meshes and Gd accumulation in selected tissues, liver, kidneys, skin and bone samples of Group 1 and Control Group were also explanted. For skin and bone samples, sites of sampling were chosen in the closest vicinity to the implanted meshes (above the implanted mesh at the surgery site for skin samples, piece of the most adjacent rib for bone samples). Three additional female Wistar rats (Charles River Laboratories) were used as control for gadolinium accumulation following intra venous injection of $0.2 \mathrm{ml} / \mathrm{kg}$ of $0.5 \mathrm{mmol} / \mathrm{ml}$ gadoteric acid (Dotarem $\AA$ ) as used in clinic (Group 2). They were then sacrificed after 1-month post-injection before explantation of liver, kidneys, skin and bone samples (in the same locations as for Group 1). All organs were degraded by exposure for 2 days to $65 \% \mathrm{HNO}_{3}$ at $100{ }^{\circ} \mathrm{C}$. The resulting residues were dissolved in $10 \mathrm{~mL}$ of diluted $\mathrm{HNO}_{3}$ and Gd was quantified by ICP-MS. 


\subsection{In vivo MRI imaging}

In vivo ${ }^{1} \mathrm{H}-\mathrm{MRI}$ investigations were performed on a 9.4 T Agilent DD2 MRI scanner operating at a frequency of $400 \mathrm{MHz}$ with GC156/100 gradients (400 mT/m) and a Quadrature Volume Coil showing a $63 \mathrm{~mm}$ internal diameter. In order to follow up the implant, longitudinal study was done at 1, 2 and 3 weeks after implantation. For each time point rats were anesthetized using isoflurane (induction at $4 \%$ and experiment around 2\%) and monitored using MR compatible small animal monitoring and Gating System (Minerve Siemens A.G., Erlangen, Germany) / RS2D (Haguenau, France). Respiration was maintained about 50 breath/min by adjusting isoflurane level and oxygen flow rate. In order to facilitate localization and analysis a water reference was put on the opposite side of the implant. Gradient echo MRI pulse sequences synchronized with the respiration in order to reduce motion artefacts (breathing period was about $1.2 \mathrm{~s}$ ) were optimized for the case of in vivo study in order to reveal the fast relaxing tissues around the IMD and by keeping an experimental duration about 15'. The following parameters were selected: repetition time $(\mathrm{TR})=1500 \mathrm{~ms}$; echo time $(\mathrm{TE})=12 \mathrm{~ms}$; Flip angle $=90 ; \mathrm{AVG}=1 ; \mathrm{FOV}=120 \mathrm{~mm} \times 60 \mathrm{~mm} ; 6$ slices; thickness: $0.5 \mathrm{~mm} ;$ gap $=0 \mathrm{~mm}$; acquisition matrix $\left(\mathrm{N}_{\mathrm{READ}} \times \mathrm{N}_{\mathrm{PHASE}}\right)=1024 \times 512$; inversion time $(\mathrm{TI})=50 \mathrm{~ms}$. All MRI processing and visualization were done using homebuilt programs under Matlab environment and ImageJ.

\section{Results and discussion}

\subsection{Preparation of $G d-D O T A-N_{3}$ and surface modification}

Aryl-azides are well known for their ability to generate highly reactive nitrenes leading to insertion into $\mathrm{CH}$ bonds and thus into polymer backbones. We recently presented this chemistry to furnish polymer substrate with antibacterial properties. ${ }^{32}$ Here, we adapted the same grafting onto method to functionalize polymer substrates with Gd-DOTA complexes (Figure 1). The ligand DOTA was chosen for its high complexation affinity with Gd. To introduce the photoreactive moiety into DOTA a simple amidation reaction between DOTA-GA anhydride and 4-azidoaniline hydrochloride was utilized. Reaction was confirmed by ${ }^{1} \mathrm{H}$ NMR with appearance of peaks at 7.6 and $7.0 \mathrm{ppm}$ corresponding to the aromatic protons of aryl-azide group and at $7.3 \mathrm{ppm}$ corresponding to the amide proton (Figure S1). Comparison between the intensity of the aromatic signals and the signals in the range 1.95-2.15 ppm corresponding to the methylene group of the spacer confirmed a $70 \%$ yield for amidation. ${ }^{13} \mathrm{C}$ NMR spectrum 
also proved the formation of DOTA-N $\mathrm{N}_{3}$ with the coexistence of the two isomers $\mathbf{1}$ and $\mathbf{2}$ shown in Figure 1 whose presence was confirmed by the peaks at 61 and 64 ppm corresponding to the methine groups and the peaks at 173 and 174 ppm corresponding to the amide groups of 2 and 1, respectively (Figure S2). TOF-MS analysis also confirmed the formation of DOTA-N 3 with a peak at 593.27 $\mathrm{Da}\left[\mathrm{M}+\mathrm{H}^{+}\right]$.

Interestingly, while DOTA is known for its quantitative complexation of Gd $\left.{ }^{35-36}\right]$, we found that the modification of DOTA with aryl azide reduced its complexation efficacy significantly, despite all coordination sites still being available. Although multiple parameters of the complexation reaction have been varied, the efficacy of Gd complexation only ranged between 14-30\% (Table S1). This rather low complexation efficacy might be explained by the presence of the isomer 2 that presents three carboxylic groups similar to the isomer 1, ie. close to the cycle, but a fourth one that is further distant by two methylene groups, which may decrease the overall complexation efficiency of the DOTA-N $\mathrm{N}_{3}$ isomer mixture. The lower complexation efficacy could also be explained low by the substituent used in this work as complexation of Gd on DOTA-GA derivatives is known to be strongly influenced by the nature of the substituent. DOTA-GA derivatives similar to ours, i.e. with an amide bond linked to the DOTA cycle by two methylene groups, are reported to be less favorable structure for Gd complexation due to both the lower value of the first protonation constant and by the weaker interaction between the amide $\mathrm{O}$-atom and $\mathrm{Gd}^{3+}$ ion associated with the formation of a less stable sixmembered chelate ring. ${ }^{37}$ In our case, the additional presence of the aromatic ring, that may hinder the motion towards a stable chelate ring, and the possible influence of the azide group could further explain the limited complexation efficacy encountered

Gd-DOTA-N ${ }_{3}$ with the highest Gd content (30\%) was further used to functionalize polymer substrates without further purification as we demonstrated in our previous work $^{32}$ that hydrophilic molecules that are not functionalized by aryl-azide moieties cannot be attached to polymer substrates under the conditions used and are removed by the washing procedures. Plates made of PLA and PP of medical grade were selected as model surfaces. Conditions for the surface photoinsertion of Gd-DOTA-N 3 were identical for both polymers and consisted in 5 sequences of spray coating of methanolic solution of Gd-DOTA-N $\mathrm{N}_{3}$, UV irradiation at 254 $\mathrm{nm}$ and subsequent rinsing of the non-anchored Gd-DOTA-N ${ }_{3}$. The efficiency of Gd-DOTA$\mathrm{N}_{3}$ immobilization was confirmed via MRI-imaging experiments and, for implanted materials, by ICP-MS analyses (see section 3.3.1). 


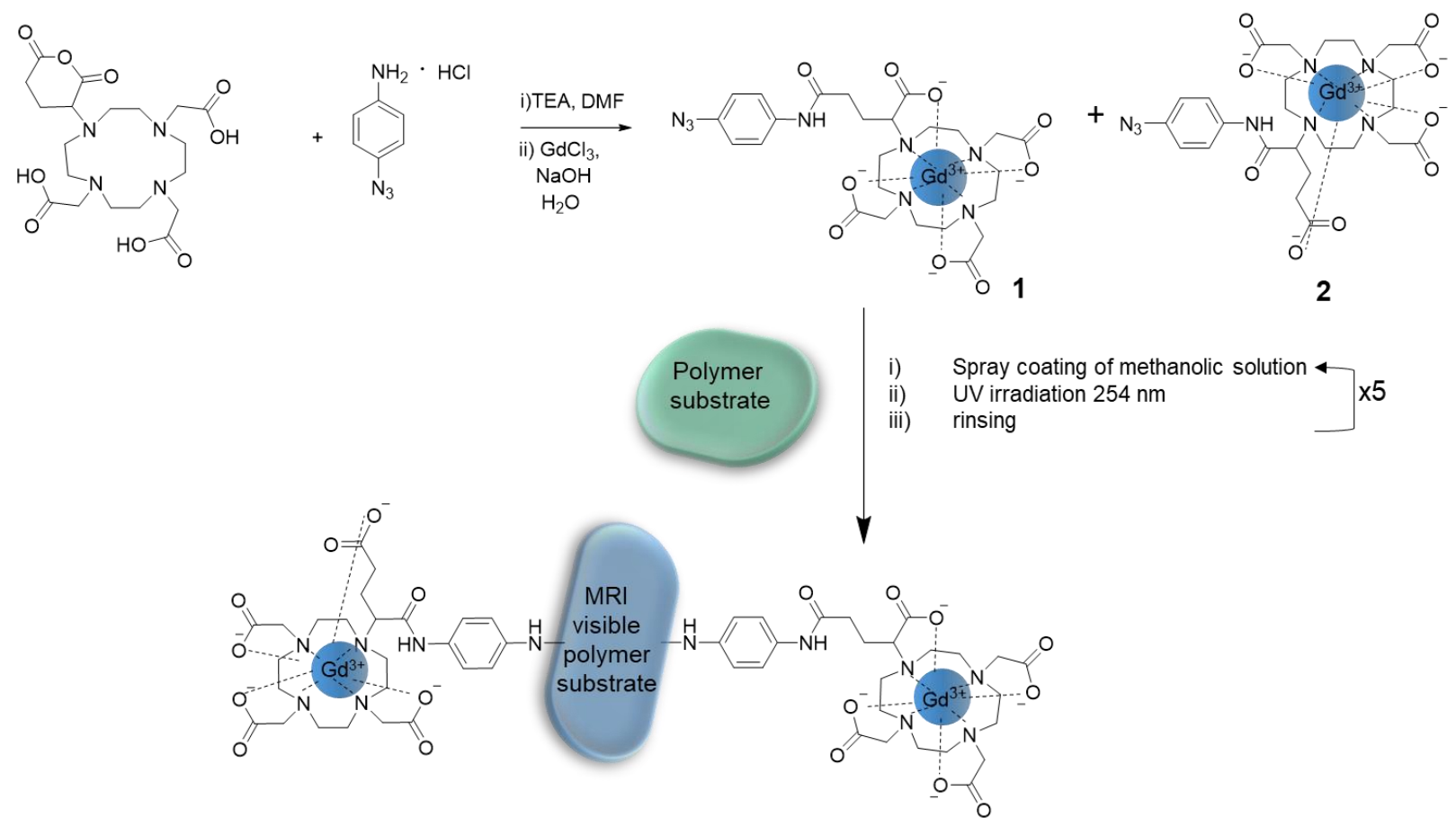

Figure 1. Synthesis of $G d-D O T A-N_{3}$ and immobilization by photoinsertion on polymer substrates.

\subsection{Biocompatibility}

Cytotoxicity of modified Gd-DOTA-N 3 PLA and PP polymers was assessed by direct contact method with L929 fibroblasts. Results were compared to non-modified PLA and PP polymers and to no polymer at all (Figure 2A). After 48h exposure, cell viability was around 96\%-100\% compared to the control without polymer. These results demonstrate that none of the Gd-DOTA functionalized polymers and their non-modified counterparts are toxic. A Kruskal-Wallis test confirmed that there is no statistical difference between the two groups $(p=0.7)$.

Additionally, biomaterials should possess the ability to support cell growth. To quantify L929 fibroblasts proliferation we measured the ability of metabolically active cells to reduce Prestoblue $^{\circledast}$ resazurin-based compound at 24, 48 and 120 h (Figure 2B). Results showed that cell proliferation is similar on pristine polymer surfaces compared to surfaces modified with Gd-DOTA-N ${ }_{3}$. There is no statistical difference between control polymer and modified GdDOTA-N3 (Mann-Whitney, $\mathrm{p}=0.9$ for PLA and $\mathrm{p}=0.5$ for PP surfaces). 
Thus, by covalently immobilizing contrast agents at the surface of PLA and PP polymers, we were able to obtain a biomaterial supporting cell growth, while presenting multifunctionality, namely preserved bulk properties (e.g. mechanical properties) and MRI visibility.
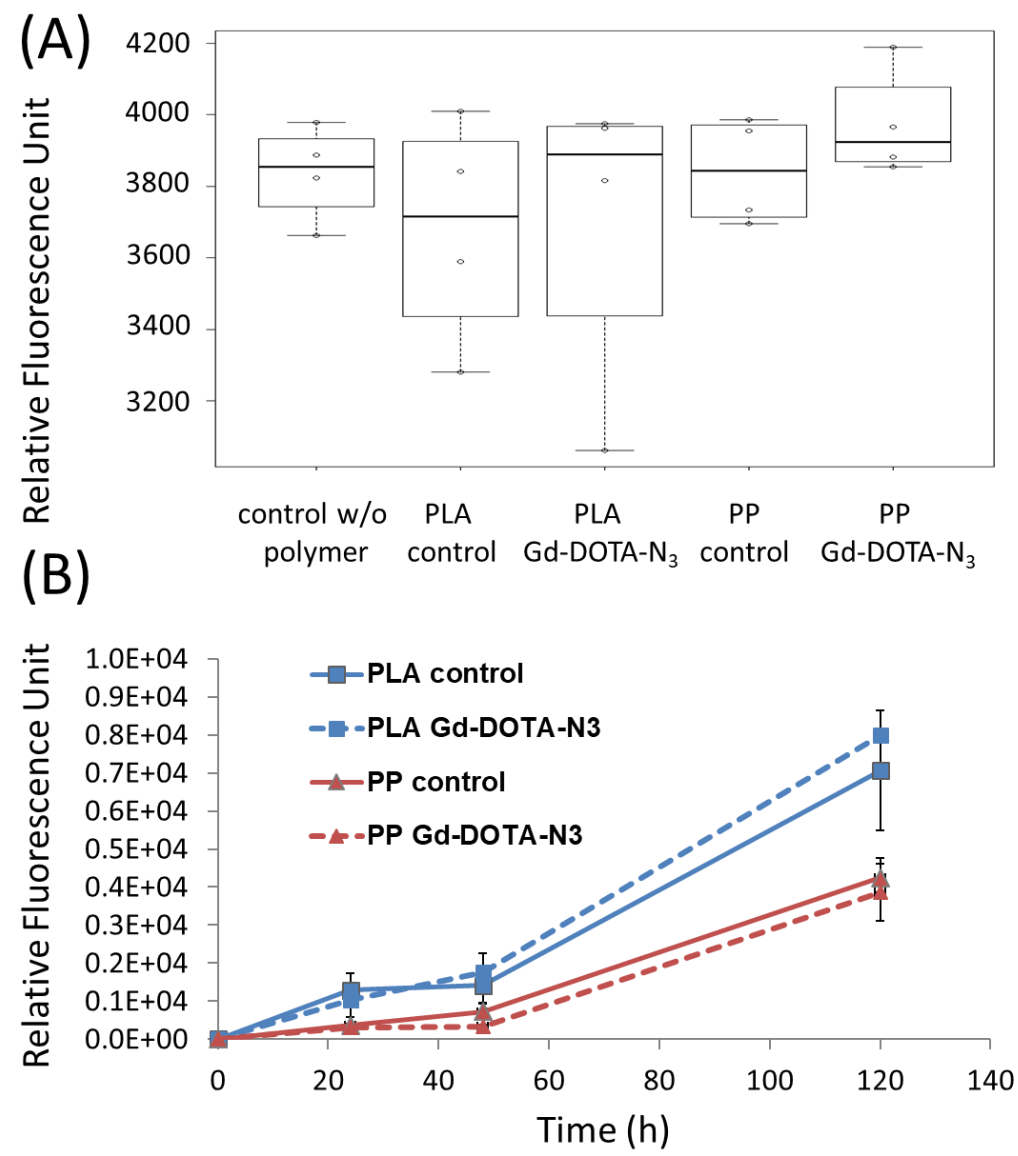

Figure 2. Cytocompatibility of polypropylene (PP) and polylactide (PLA) modified with Gd-DOTA-N3. (A) Cytotoxicity effects of polymer on L929 cells after $48 \mathrm{~h}$ evaluated by PrestoBlue ${ }^{\circledR}$ assay (data are expressed as median and interquartile range with maximum and minimum data points , $\mathrm{n}=4$ ); and (B) L929 proliferation on modified surfaces compared to pristine surfaces at 1,2 and 5 days (data are expressed as means \pm SD and correspond to measurements with $n=4$ ). (For sake of clarity TC controls compared to other surfaces are provided as supplementary data in Figure S3).

\subsection{In vitro MRI-imaging}

\subsubsection{MRI visibility of polymeric substrates}

In order to verify the versatility of the methodology to render most polymeric IMD visible by MRI, we selected a variety of polymer largely used in fabricating MD. In addition to PLA and PP, plates made of PEEK and PU were modified as previously described. All plates were then embedded in agarose gel for MRI experiments. As shown in Figure 3A, all polymers modified 
with Gd-DOTA-N ${ }_{3}$ were visible using MRI spin echo sequence. PP and PU were more contrasted compared to PEEK and PLA. This observation is in agreement with our previous work, where in an effort to generate antibacterial polymer surfaces, we witnessed some variability in terms of insertion efficiency depending on the polymer substrate. This was attributed to the ability of the aryl-azide bearing (macro)molecules to interact with the surface, which depends strongly on the respective hydrophilicity/hydrophobicity of the two entities. ${ }^{32}$ It is therefore our belief that the difference of MRI signal intensity observed here are due to the variability of chemical affinity between the polymer substrates and the Gd-DOTA-N ${ }_{3}$.

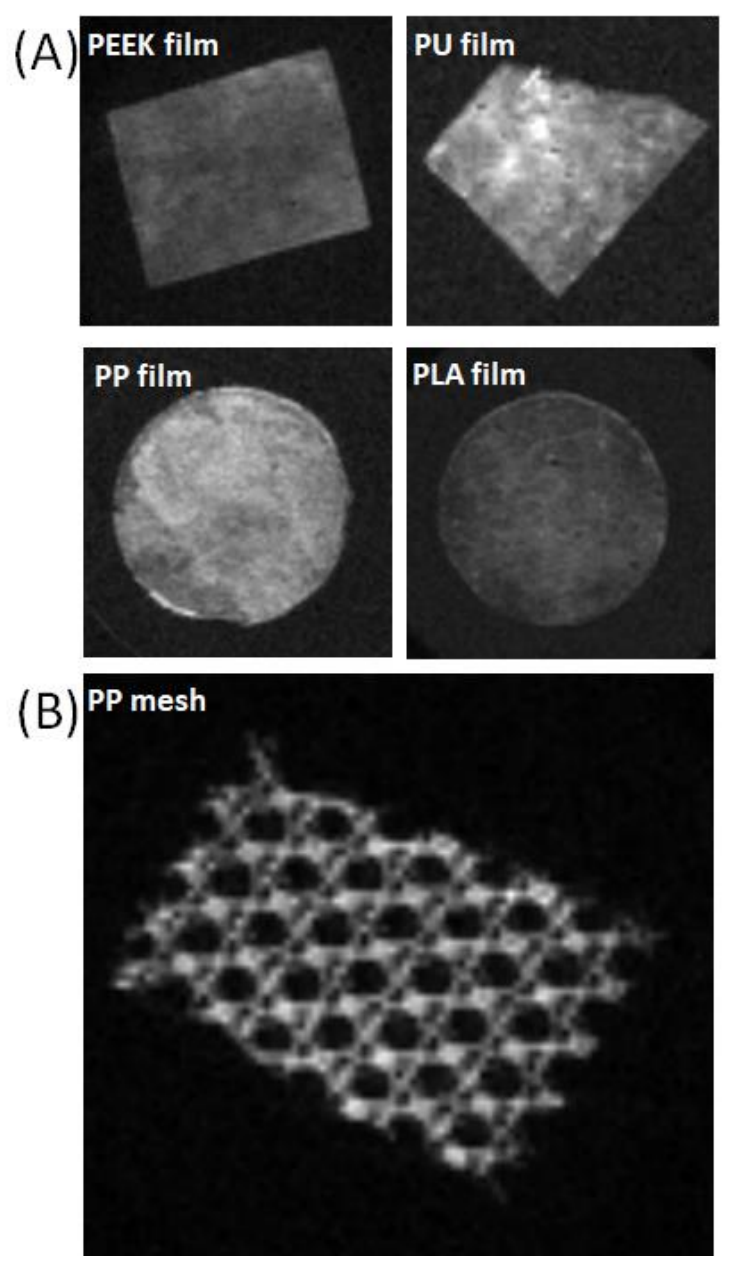

Figure 3. MRI visualization of polymer surfaces modified with Gd-DOTA-N3. Various polymers relevant in the frame of medical devices field are visualized by MRI (7T) following their surface modification with Gd-DOTA-N 3 . (A) poly(ether ether ketone), polyurethane, polypropylene and polylactide plates.(B) polypropylene surgical mesh.

Following the modification of PP plates, we also assessed the modification of PP meshes that are among the most largely implanted medical devices. ${ }^{38}$ Such surgical meshes are in fact used for the treatment of hernia as well as in procedures aimed at treating pelvic organ prolapse. 
Numerous recent studies focused therefore on the improvement of this type of MD by making them trackable using MRI to follow their morphology (contraction) and position (folding, migration) changes. ${ }^{20-21,39-40}$ The PP meshes were modified using the same procedure as the one used for the plates. ICP-MS analyses confirmed the surface functionalization with a concentration of Gd of $73 \mathrm{ng} / \mathrm{mg}$ of mesh. Considering the areal density $\left(72 \mathrm{~g} / \mathrm{m}^{2}\right)$, the surface of the meshes $\left(1.91 \mathrm{~m}^{2} / \mathrm{m}^{2}\right)$ and the complexation efficacy this value corresponds to a grafting density of $3.3 \mathrm{nmol} / \mathrm{cm}^{2}$ of $\mathrm{Gd}^{3+}$ and $11 \mathrm{nmol} / \mathrm{cm}^{2}$ of Gd-DOTA-N 3 . In vitro MRI confirmed the good visibility of the modified PP mesh with a strong hypersignal obtained under the conditions selected for the spin echo sequence (Figure 3B).

\subsubsection{Persistence of MRI visibility of PP meshes}

Prior to in vivo tests, detectability and stability of the surface modification, and therefore of the imaging properties of the modified meshes were assessed over 12 months. For this, agarose gels embedding the modified meshes were stored over one year at $4^{\circ} \mathrm{C}$. Compared MRI-visibility at 0 and 1 year are shown in Figure 4A. Using MATLAB programming language the signal surrounding the implant was isolated allowing for an image treatment (Figure 4B), and determination of the intensity of the signal to noise ratio (SNR) from meshes. 


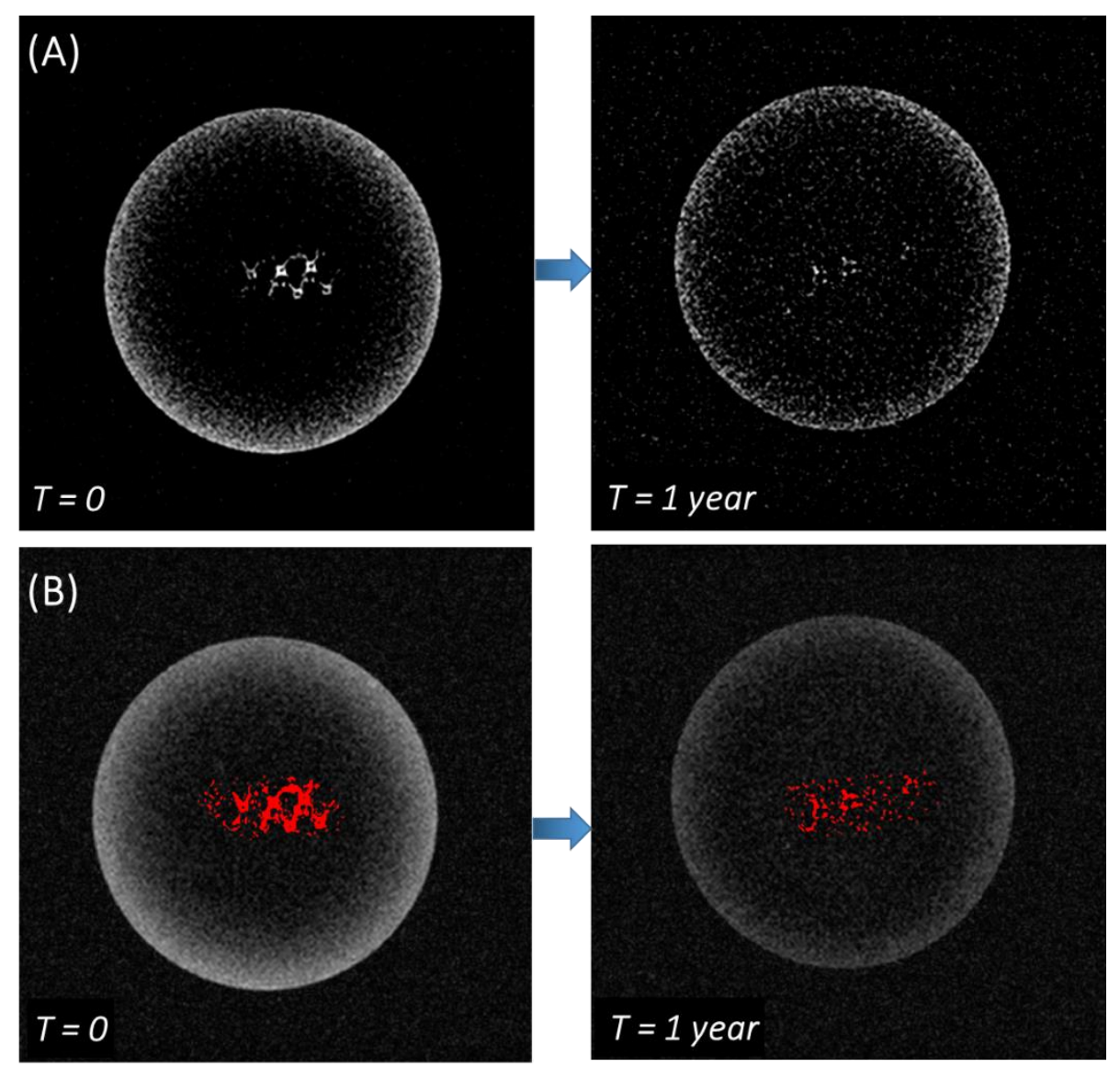

Figure 4. In vitro MRI visualization of modified PP meshes. T1-weighted 2D spin echo images were acquired using 9.4T Agilent horizontal bore scanner. (A) Stability study over one year using the raw signal. (B) Stability study over one year using the intensity of the signal to noise ratio (SNR).

Although a clear SNR decrease (70\%) was witnessed over the one-year storage period (Figure 5A), the modified PP mesh was still detectable. This SNR decrease could be due to partial release of Gd from the chelate. In fact, despite the use of DOTA as chelate, its modification may have led to a decrease of complexation constant that is highly sensitive to the chelate molecular structure. ${ }^{41-43}$. More in-depth study of this aspect was beyond the scope of the present work but should be investigated in the future. 

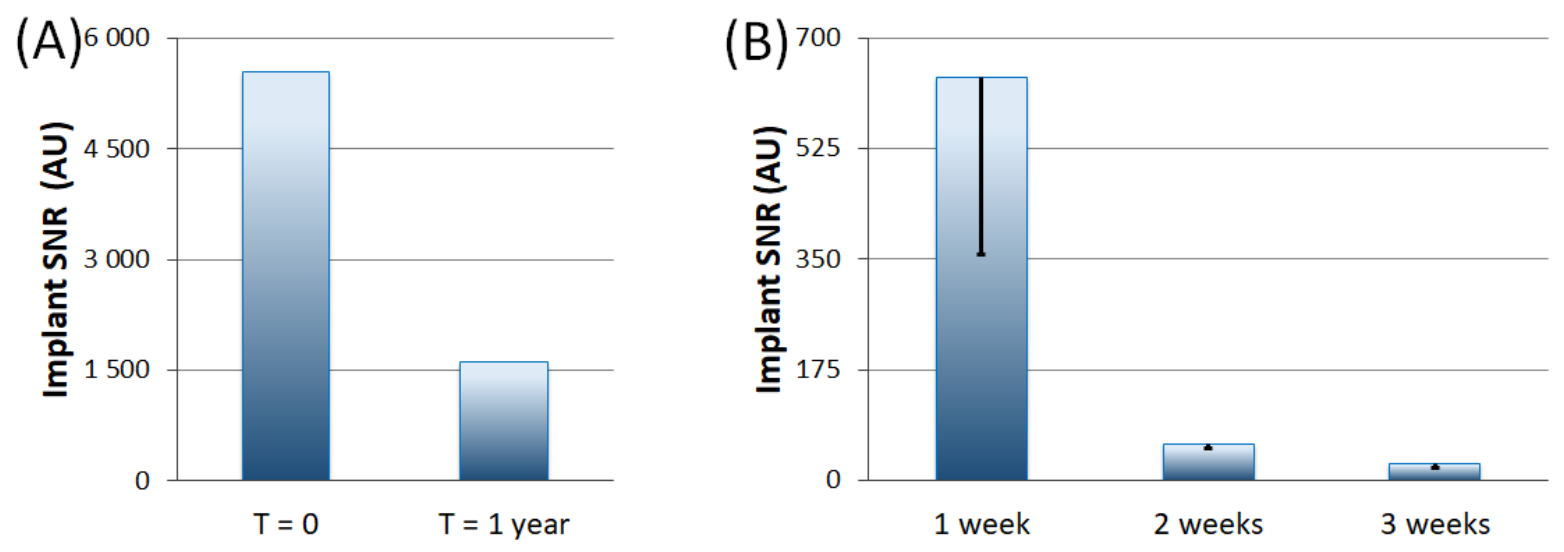

Figure 5. Intensity of the signal to noise ratio (SNR) from meshes. T1-weighted 2D spin echo images were acquired using 9.4T Agilent horizontal bore scanner. (A) in vitro stability study over one year (single measurement). (B) in vivo stability study over three weeks (data are expressed as means \pm SD and correspond to measurements with $n=3$ )

\subsection{In vivo MRI-imaging}

In vivo visibility and stability was evaluated by T1-weighted MRI at 9.4T and performed at different time points after the implantation of PP meshes in rats. Each rat was implanted with a control mesh and a MRI-visible modified mesh (Figure 6A). The same batch of PP meshes used for in vitro evaluations was implanted. Whereas pristine PP meshes were not detected, PP meshes modified with Gd-DOTA-N 3 were clearly visible at 1-week post-implantation. In more det, under the selected in vivo MRI conditions, modified PP-mesh appeared in hyposignal within a region of hypersignal (Figure 6B). This hypersignal was attributed to the presence of the Gd at the surface of the mesh interacting with the surrounding water molecules, and to a minor inflammation contribution. This second contribution is also seen in the area of the nonmodified control mesh, but as expected the absence of Gd did not allow to visualize the control mesh. Following a treatment of the data with MATLAB (procedure developed at the BioNanoImaging Foundry user facility), results show a strong signal enhancement in normalized SNR for the modified PP-meshes 1 week after implantation, which decreased rapidly in time (Figure 6C). After 2 weeks SNR was only $10 \%$ compared to 1 week, and $4 \%$ after 3 weeks of implantation (Figure 5B). Similar to the in vitro observations, a significant decrease of visibility is witnessed in vivo at an even faster rate. This rapid loss of signal might be due to the presence of metals that have high affinity for the Gd-binding ligand like $\mathrm{Zn}^{2+}$, $\mathrm{Cu}^{2+}$, or $\mathrm{Fe}^{3+}$ and/or the presence of ligands with high affinity for Gd such as phosphate and 
carbonate. However, these in vivo experiments confirmed the potential of the proposed strategy that allows for the MRI visualization of commercial off-the-shelf medical devices.
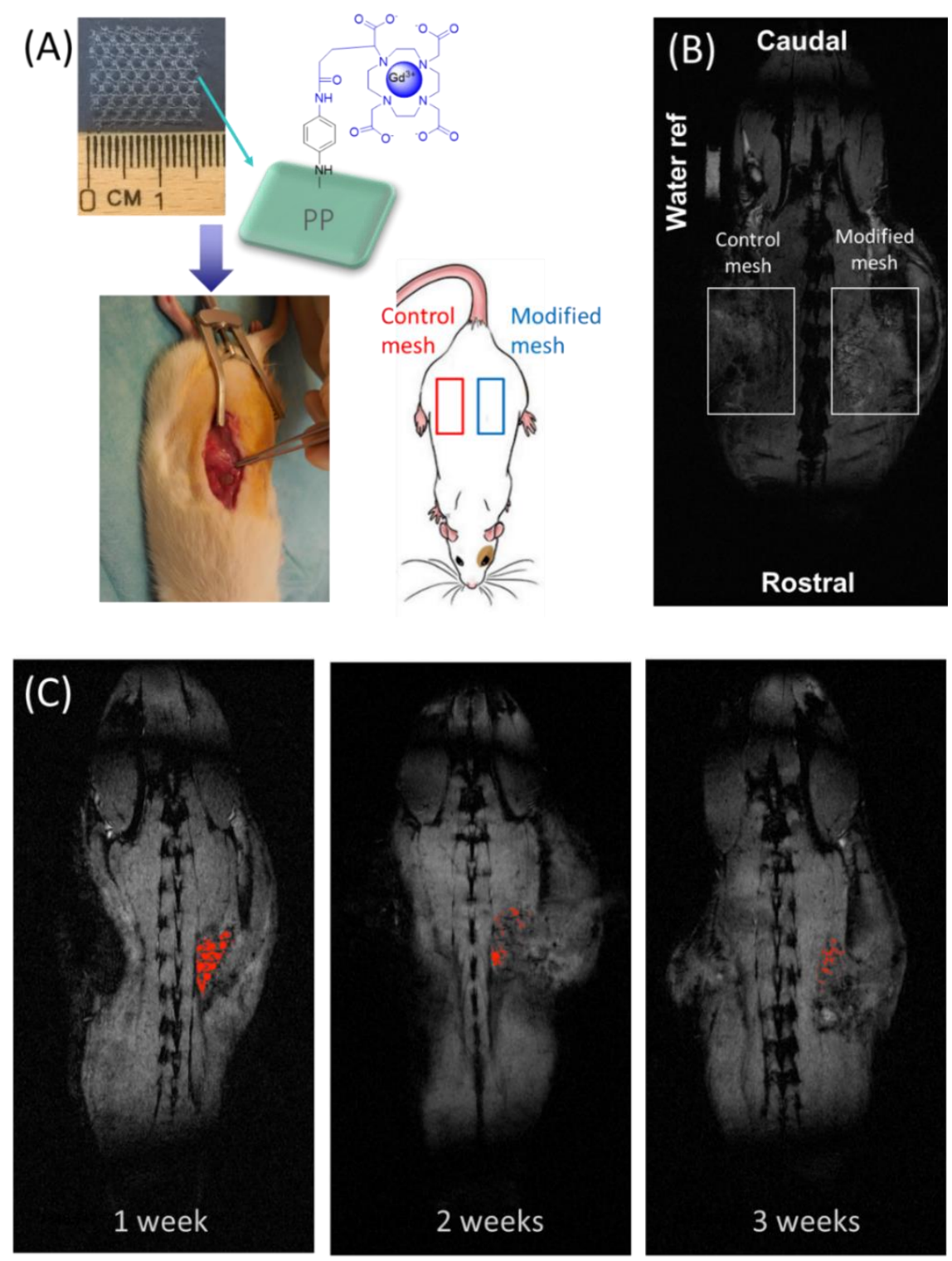

Figure 6. In vivo MRI visualization of modified PP meshes. (A) Intermuscular lumbar muscle implantation of control and modified meshes. (B) Typical native MRI signal at 1 week post-implantation (water reference is visible on the top left corner). (C) Visibility of the modified mesh over 3 weeks using the intensity of the signal to noise ratio (SNR).

\subsection{Histopathological evaluation}

Scaffold integration was evaluated by microscopic staining of the HES slides after a 1-month dorsal implantation into rats (Figure 7A-C). Meshes threads appeared as a white elongated regular material on the histological sections (black arrows). We observed minimal cicatricial fibrosis (green arrows) associated with a moderate cellular inflammatory reaction around meshes (blue arrow). Presence of macrophages and multinucleated giant cells were observed, 
characteristics of a classic foreign body reaction. Grading of the inflammation was carried out in terms of intensity and spreading (Figure 7D). Tissues at the surgical site of rats having received the same surgical procedure but without any mesh implantation were used as control to determine the absence of inflammatory zone and of lesion spreading (level 0 ). There is no microscopic difference between pristine and modified PP meshes regarding to lesions intensity and spreading.

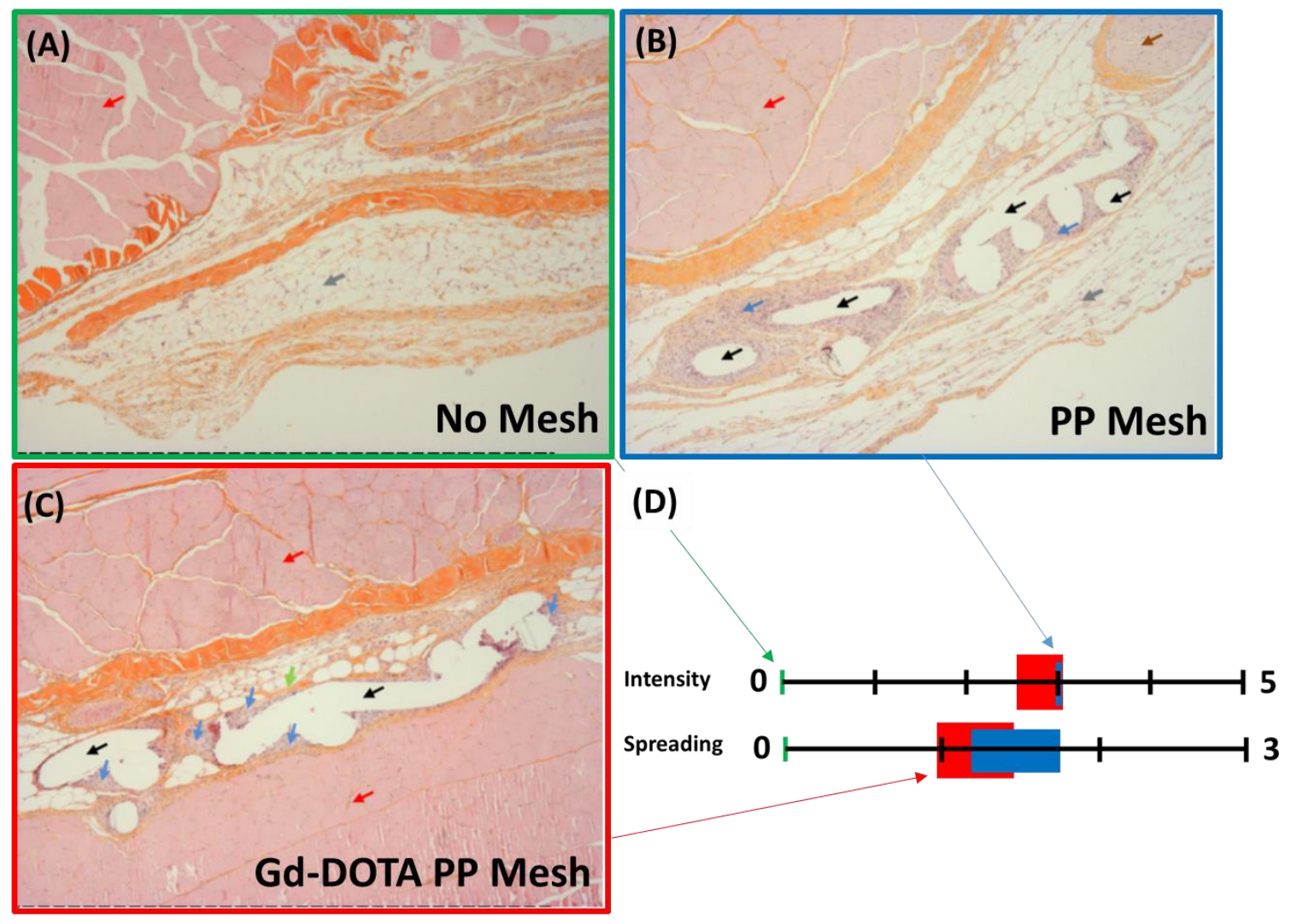

Figure 7. Histological evaluation of PP mesh and PP-mesh modified with Gd-DOTA-N3 (images were captured using $5 \times$ magnification by light microscopy). (A, B, C) typical histological section (HES, x5) with muscle tissues (red arrows), fat tissue (grey arrows), mesh (black arrows), inflammation (blue arrows), and fibrosis (green arrows). (D) Grading of inflammation in implanted rats $(n=4)$. (A) Control tissues at the surgical site of rats having received the same surgical procedure but without any mesh implantation; (B) tissues surrounding non-modified PP meshes; and (C) tissues surrounding the Gd-DOTA-N 3 modified PP meshes.

\subsection{Biodistribution of $\mathrm{Gd}$}

To evaluate the stability of Gd immobilization at the surface of the PP meshes, Gd biodistribution was assessed at 1-month post-implantation. Following the sacrifice of rats implanted with modified meshes (Group 1), liver, kidneys, skin and bone samples were explanted. For skin and bone samples, sites of sampling were chosen in the closest vicinity to 
the implanted meshes (cf. experimental part). After mineralization of the tissues, ICP-MS analyses were carried out to quantify their Gd content. Tissues from rats having surgery without mesh implantation have been used as negative controls (Supp data Figure S4). In parallel, concentrations of $\mathrm{Gd}$ were also assessed in tissues from rats having received only a bolus injection of the clinical contrast agent Dotarem ${ }^{\circledR}$ (Guerbet, France) at the dose prescribed by the supplier and sacrificed also at 1-month post-injection (Group 2). As expected, Gd mostly accumulated in the kidneys at a dose of $0.37 \mathrm{ng} / \mathrm{mg}$ of organ after the bolus injection. The other tissues only displayed marginal concentrations of Gd (Figure 8 inset). In contrast, Gd released from the mesh accumulated in all tested tissues but at low doses (Figure 8). The highest concentration was found in skin $(0.15 \mathrm{ng} / \mathrm{mg}$ of organ $)$, followed by kidneys $(0.09 \mathrm{ng} / \mathrm{mg}$ of organ), bone (0.05 ng/mg of organ) and liver (0.02 ng/mg of organ). The cumulated concentration of Gd released from the modified mesh was of $0.31 \mathrm{ng} / \mathrm{mg}$ of organ, which is in the same range as the value found for the bolus injection. This is surprising considering the 4000 times lower dose of Gd present on the implanted meshes (800 ng of Gd per mesh as quantified by ICP-MS) compared with the dose of Gd injected using Dotarem® (3 mg of Gd per rat).

This result highlights the impact of the formulation and location of the Gd source. Although high doses of $\mathrm{Gd}$ are injected when using Dotarem ${ }^{\circledR}$ clinical formulations, it is rapidly eliminated with a terminal halflife for blood elimination of about 1.5 hours when administered to subjects with normal renal function. ${ }^{44}$ In addition, being almost exclusively eliminated via the kidney it mainly accumulates in this organ, and only marginally accumulates in other organs and tissues (mainly liver and bone). ${ }^{45}$ In contrast, despite low initial amounts of Gd on the modified meshes, its partial release in the surrounding tissues strongly modifies its biodistribution. In particular, it is found in cutaneous samples that are adjacent to the surgical site (skin explants were taken from the site of surgery), which in the case of bolus injection of Dotarem ${ }^{\circledR}$, is only observed in skin biopsies of NSF patients, although a recent study also demonstrated the possibility of Gd deposition in the skin of a patient with normal renal function. ${ }^{46}$ In our case, the difference observed in terms of biodistribution are likely related to the physiological conditions found at the site of injection/implantation (e.g. transmetallation ${ }^{43}$ ). It is also reported that several classes of proteins act as potential hosts for the $\mathrm{Gd}^{3+}$ ion such as $\mathrm{Ca}^{2+}$-binding proteins, which constitute one of the largest class of metalloproteins present in the extracellular and intracellular space or glycosaminoglycans, which are components of the extracellular matrix, for example, in the skin and bones, and have strong complexing ability. ${ }^{47-}$ 
${ }^{48}$ It is therefore expected that the biodistribution of Gd strongly differs when injected in blood or implanted in conjunctive tissues. This finding further demonstrates that in the frame of MRIvisible medical devices, it is of prime importance to strongly reduce the overall amount of contrast agent immobilized at the surface.

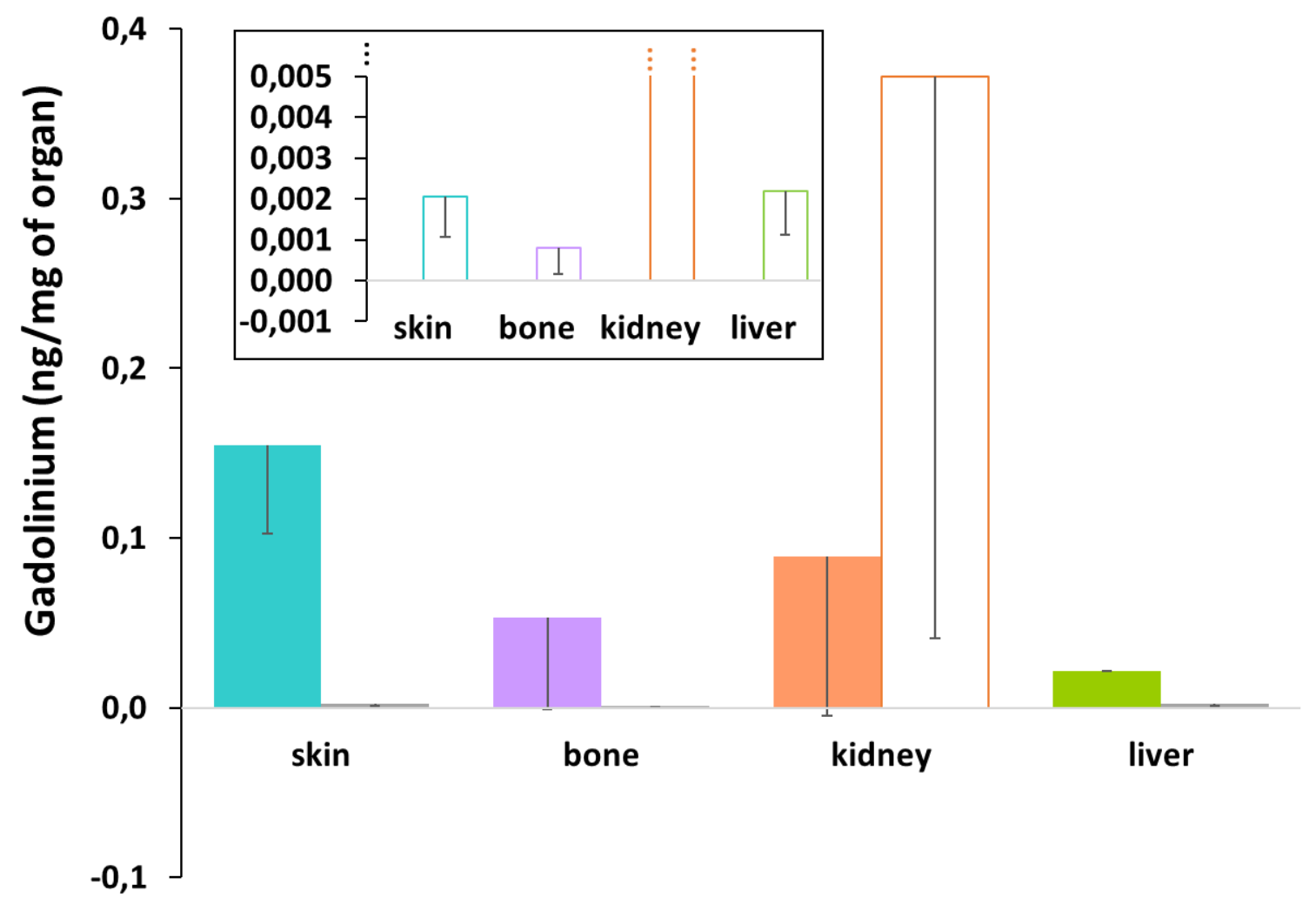

Figure 8. Biodistribution of gadolinium: mesh implantation vs. bolus injection. Concentration of $\mathrm{Gd}$ in the organs of rats implanted with meshes modified with Gd-DOTA-N 3 (plain bars, Group 1) at 1-month post-implantation, and of rats having received a bolus injection of Dotarem ${ }^{\circledR}$ (empty bars, Group 2) at 1-month post-injection (data are expressed as means \pm $\mathrm{SD}$ and correspond to measurements with $\mathrm{n}=4$ for Group 1, and $\mathrm{n}=3$ for Group 2). Inset corresponds to a zoom of the y axis to visualize the biodistribution of Gd in skin, bone and liver for Group 2.

\section{Conclusion}

Rendering polymeric biomaterials MRI-visible by utilizing the photomodification with aryl azide modified Gd-DOTA complexes is a straight forward and very easy to implement method. However, the introduction of the photoreactive moiety into the DOTA ligand structure, impedes its complexation efficacy towards Gd significantly. While visibility is initially sufficiently high, the effect is only medium term. To ensure high contrast long term, there is still need for future development to further increase the stability of the Gd-complex. Nevertheless, the approach 
proposed in this work appears as an attractive mean to easily yield MRI-visibility while guaranteeing low doses of contrast agents.

\section{Acknowledgements}

The authors wish to thank University of Montpellier (UM1 7th Postdoctoral Fellowship Program) and French Embassy in Germany-Department for Science (French-German Post-doctoral action 2015) for A.S.'s fellowship

\section{References}

[1]. Systematic review of needs for medical devices for ageing populations; World Health Organization: 2015.

[2]. Sullivan, F., Global Medical Device Market Outlook 2018. In AusMedTech, 2018.

[3]. Beal, M. W., Interventional Management of Urethral Obstructions. Vet. Clin. North Am. Small Anim. Pract. 2018, 48 (5), 863-874.

[4]. Behar, J. M.; Claridge, S.; Jackson, T.; Sieniewicz, B.; Porter, B.; Webb, J.; Rajani, R.; Kapetanakis, S.; Carr-White, G.; Rinaldi, C. A., The role of multi modality imaging in selecting patients and guiding lead placement for the delivery of cardiac resynchronization therapy. Expert Rev. Cardiovasc. Ther. 2017, 15 (2), 93-107.

[5]. Campbell-Washburn, A. E.; Tavallaei, M. A.; Pop, M.; Grant, E. K.; Chubb, H.; Rhode, K.; Wright, G. A., Real-time MRI guidance of cardiac interventions. J. Magn. Reson. Imaging 2017, 46 (4), 935-950.

[6]. Marcus, H. J.; Cundy, T. P.; Nandi, D.; Yang, G. Z.; Darzi, A., Robot-assisted and fluoroscopyguided pedicle screw placement: a systematic review. Eur. Spine J. 2014, 23 (2), 291-7.

[7]. Vogl, T. J.; Tawfik, A.; Emam, A.; Naguib, N. N.; Nour-Eldin, A.; Burck, I.; Stover, T., Pre-, Intraand Post-Operative Imaging of Cochlear Implants. RoFo 2015, 187 (11), 980-9.

[8]. Cerato, A.; Luyckx, M.; Ghaye, B., Migration of implanon contraceptive implant into the pulmonary artery. Diagn. Interv. Imaging 2018, In press.

[9]. Harrison, S. C.; Winterbottom, A. J.; Coughlin, P. A.; Hayes, P. D.; Boyle, J. R., Editor's Choice Mid-term Migration and Device Failure Following Endovascular Aneurysm Sealing with the Nellix Stent Graft System - a Single Centre Experience. Eur. J. Vasc. Endovasc. Surg. 2018, 56 (3), 342-348.

[10]. Harsløf, S. S.; Wara, P.; Friis-Andersen, H., Fixation devices in laparoscopic ventral hernia repair: a review. Surg. Technol. Int. 2014, 24, 203-213.

[11]. Chapple, C. R.; Cruz, F.; Deffieux, X.; Milani, A. L.; Arlandis, S.; Artibani, W.; Bauer, R. M.; Burkhard, F.; Cardozo, L.; Castro-Diaz, D.; Cornu, J. N.; Deprest, J.; Gunnemann, A.; Gyhagen, M.; Heesakkers, J.; Koelbl, H.; MacNeil, S.; Naumann, G.; Roovers, J. W. R.; Salvatore, S.; Sievert, K. D.; Tarcan, T.; Van der Aa, F.; Montorsi, F.; Wirth, M.; Abdel-Fattah, M., Consensus Statement of the European Urology Association and the European Urogynaecological Association on the Use of Implanted Materials for Treating Pelvic Organ Prolapse and Stress Urinary Incontinence. Eur. Urol. 2017, 72 (3), 424-431.

[12]. Nottelet, B.; Darcos, V.; Coudane, J., Aliphatic polyesters for medical imaging and theranostic applications. Eur. J. Pharm. Biopharm. 2015, 97, 350-370.

[13]. Anton, N.; Vandamme, T. F., Nanotechnology for computed tomography: a real potential recently disclosed. Pharm. Res. 2014, 31, 20-34.

[14]. Marcos, M.; Cano, P.; Fantazzini, P.; Garavaglia, C.; Gomez, S.; Garrido, L., NMR relaxometry and imaging of water absorbed in biodegradable polymer scaffolds. Magn. Reson. Imaging 2006, 24, 89-95. 
[15]. Ramalho, J.; Semelka, R. C.; Ramalho, M.; Nunes, R. H.; AlObaidy, M.; Castillo, M., GadoliniumBased Contrast Agent Accumulation and Toxicity: An Update. AJNR Am. J. Neuroradiol. 2016, 37 (7), 1192-8.

[16]. Kanda, T.; Fukusato, T.; Matsuda, M.; Toyoda, K.; Oba, H.; Kotoku, J.; Haruyama, T.; Kitajima, K.; Furui, S., Gadolinium-based Contrast Agent Accumulates in the Brain Even in Subjects without Severe Renal Dysfunction: Evaluation of Autopsy Brain Specimens with Inductively Coupled Plasma Mass Spectroscopy. Radiology 2015, 276 (1), 228-32.

[17]. Agency, E. M. Gadolinium-containing contrast agents; European Medicines Agency: 2017.

[18]. Franconi, F.; Roux, J.; Garric, X.; Lemaire, L., Early Postsurgical Visualization of Composite Mesh Used in Ventral Hernia Repair by Amide Proton Transfer MRI. Magn. Reson. Med. 2014, 71 (1), 313317.

[19]. Boehm-Sturm, P.; Haeckel, A.; Hauptmann, R.; Mueller, S.; Kuhl, C. K.; Schellenberger, E. A., Low-Molecular-Weight Iron Chelates May Be an Alternative to Gadolinium-based Contrast Agents for T1-weighted Contrast-enhanced MR Imaging. Radiology 2018, 286 (2), 537-546.

[20]. Endo, M.; Feola, A.; Sindhwani, N.; Manodoro, S.; Vlacil, J.; Engels, A. C.; Claus, F.; Deprest, J. A., Mesh contraction: in vivo documentation of changes in apparent surface area utilizing meshes visible on magnetic resonance imaging in the rabbit abdominal wall model. Int. Urogynecol. J. 2014, 25 (6), 737-43.

[21]. Otto, J.; Kuehnert, N.; Kraemer, N. A.; Ciritsis, A.; Hansen, N. L.; Kuhl, C.; Busch, D.; Peter Neumann, U.; Klinge, U.; Conze, K. J., First in vivo visualization of MRI-visible IPOM in a rabbit model. J. Biomed. Mater. Res. B Appl. Biomater. 2014, 102 (6), 1165-9.

[22]. Peng, H.; Blakey, I.; Dargaville, B.; Rasoul, F.; Rose, S.; Whittaker, A. K., Synthesis and Evaluation of Partly Fluorinated Block Copolymers as MRI Imaging Agents. Biomacromolecules 2009, 10 (2), 374381.

[23]. Tirotta, I.; Dichiarante, V.; Pigliacelli, C.; Cavallo, G.; Terraneo, G.; Bombelli, F. B.; Metrangolo, P.; Resnati, G., (19)F magnetic resonance imaging (MRI): from design of materials to clinical applications. Chem. Rev. 2015, 115 (2), 1106-29.

[24]. Lammers, T.; Mertens, M. E.; Schuster, P.; Rahimi, K.; Shi, Y.; Schulz, V.; Kuehne, A. J. C.; Jockenhoevel, S.; Kiessling, F., Fluorinated polyurethane scaffolds for (19)F magnetic resonance imaging. Chem. Mater. 2017, 29 (7), 2669-2671.

[25]. Zhang, C.; Moonshi, S. S.; Han, Y.; Puttick, S.; Peng, H.; Magoling, B. J. A.; Reid, J. C.; Bernardi, S.; Searles, D. J.; Král, P.; Whittaker, A. K., PFPE-Based Polymeric 19F MRI Agents: A New Class of Contrast Agents with Outstanding Sensitivity. Macromolecules 2017, 50 (15), 5953-5963.

[26]. Garmendia, S.; Mantione, D.; Alonso-de Castro, S.; Jehanno, C.; Lezama, L.; Hedrick, J. L.; Mecerreyes, D.; Salassa, L.; Sardon, H., Polyurethane based organic macromolecular contrast agents (PU-ORCAs) for magnetic resonance imaging. Polym. Chem. 2017, 8 (17), 2693-2701.

[27]. El Habnouni, S.; Nottelet, B.; Darcos, V.; Porsio, B.; Lemaire, L.; Franconi, F.; Garric, X.; Coudane, J., MRI-Visible Poly(epsilon-caprolactone) with Controlled Contrast Agent Ratios for Enhanced Visualization in Temporary Imaging Applications. Biomacromolecules 2013, 14 (10), 3626-3634.

[28]. Blanquer, S.; Guillaume, O.; Letouzey, V.; Lemaire, L.; Franconi, F.; Paniagua, C.; Coudane, J.; Garric, X., New magnetic-resonance-imaging-visible poly(-caprolactone)-based polyester for biomedical applications. Acta Biomater. 2012, 8 (3), 1339-1347.

[29]. Guillaume, O.; Blanquer, S.; Letouzey, V.; Cornille, A.; Huberlant, S.; Lemaire, L.; Franconi, F.; de Tayrac, R.; Coudane, J.; Garric, X., Permanent Polymer Coating for in vivo MRI Visualization of Tissue Reinforcement Prostheses. Macromol. Biosci. 2012, 12 (10), 1364-1374.

[30]. Younis, M.; Darcos, V.; Paniagua, C.; Ronjat, P.; Lemaire, L.; Nottelet, B.; Garric, X.; Bakkour, Y.; El Nakat, J. H.; Coudane, J., MRI-visible polymer based on poly(methyl methacrylate) for imaging applications. RSC Adv. 2016, 6 (7), 5754-5760.

[31]. Letouzey, V.; Huberlant, S.; Cornille, A.; Blanquer, S.; Guillaume, O.; Lemaire, L.; Garric, X.; De Tayrac, R., Tolerance and long-term MRI imaging of gadolinium-modified meshes used in soft organ repair. Plos One 2015, 10 (3), 1-12. 
[32]. Schulz, A.; Stocco, A.; Bethry, A.; Lavigne, J.-P.; Coudane, J.; Nottelet, B., Direct Photomodification of Polymer Surfaces: Unleashing the Potential of Aryl-Azide Copolymers. Adv. Funct. Mater. 2018, 28 (30), 1800976.

[33]. Bernhard, C.; Moreau, M.; Lhenry, D.; Goze, C.; Boschetti, F.; Rousselin, Y.; Brunotte, F.; Denat, F., DOTAGA-Anhydride: A Valuable Building Block for the Preparation of DOTA-Like Chelating Agents. Chem. Eur. J. 2012, 18 (25), 7834-7841.

[34]. Brown, R.; Clarke, D.; Daffner, R., Is a Mixture of Gadolinium and lodinated Contrast Material Safe During MR Arthrography? Am. J. Roentgenol. 2000, 175, 1087-1090.

[35]. Idée, J.-M.; Port, M.; Robic, C.; Medina, C.; Sabatou, M.; Corot, C., Role of thermodynamic and kinetic parameters in gadolinium chelate stability. J. Magn. Reson. Imaging 2009, 30 (6), 1249-1258.

[36]. Meyer, D.; Schaefer, M.; Bonnemain, B., Gd-DOTA, a potential MRI contrast agent. Current status of physicochemical knowledge. Invest. Radiol. 1988, 23 (1), S232-S235.

[37]. Tei, L.; Baranyai, Z.; Gaino, L.; Forgács, A.; Vágner, A.; Botta, M., Thermodynamic stability, kinetic inertness and relaxometric properties of monoamide derivatives of lanthanide(iii) DOTA complexes. Dalton Trans. 2015, 44 (12), 5467-5478.

[38]. Klinge, U.; Klosterhalfen, B., Mesh implants for hernia repair: an update. Expert Rev. Med. Devices 2018, 15 (10), 735-746.

[39]. Iva, U.; Nikhil, S.; Geertje, C.; Alice, T.; Rynkevic, R.; Lucie, H.; Andrew, F.; Jan, D., In vivo documentation of shape and position changes of MRI-visible mesh placed in rectovaginal septum. J. Mech. Behav. Biomed. Mater. 2017, 75, 379-389.

[40]. Lambertz, A.; van den Hil, L. C. L.; Ciritsis, A.; Eickhoff, R.; Kraemer, N. A.; Bouvy, N. D.; Mullen, A.; Klinge, U.; Neumann, U. P.; Klink, C. D., MRI Evaluation of an Elastic TPU Mesh under Pneumoperitoneum in IPOM Position in a Porcine Model. J. Invest. Surg. 2018, 31 (3), 185-191.

[41]. Laurent, S.; Henoumont, C.; Vander Elst, L.; Muller, R. N., Synthesis and Physicochemical Characterisation of Gd-DTPA Derivatives as Contrast Agents for MRI. Eur. J. Inorg. Chem. 2012, (12), 1889-1915.

[42]. Li, Y.; Laurent, S.; Esser, L.; Elst, L. V.; Muller, R. N.; Lowe, A. B.; Boyer, C.; Davis, T. P., The precise molecular location of gadolinium atoms has a significant influence on the efficacy of nanoparticulate MRI positive contrast agents. Polym. Chem. 2014, 5 (7), 2592-2601.

[43]. Frenzel, T.; Lengsfeld, P.; Schirmer, H.; Hutter, J.; Weinmann, H. J., Stability of GadoliniumBased Magnetic Resonance Imaging Contrast Agents in Human Serum at 37 degrees C. Invest. Radiol. 2008, 43 (12), 817-828.

[44]. Aime, S.; Caravan, P., Biodistribution of gadolinium-based contrast agents, including gadolinium deposition. J. Magn. Reson. Imaging 2009, 30 (6), 1259-67.

[45]. Tweedle, M. F.; Wedeking, P.; Kumar, K., Biodistribution of radiolabeled, formulated gadopentetate, gadoteridol, gadoterate, and gadodiamide in mice and rats. Invest. Radiol. 1995, 30 (6), 372-80.

[46]. Roberts, D. R.; Lindhorst, S. M.; Welsh, C. T.; Maravilla, K. R.; Herring, M. N.; Braun, K. A.; Thiers, B. H.; Davis, W. C., High Levels of Gadolinium Deposition in the Skin of a Patient With Normal Renal Function. Invest. Radiol. 2016, 51 (5), 280-9.

[47]. Taupitz, M.; Stolzenburg, N.; Ebert, M.; Schnorr, J.; Hauptmann, R.; Kratz, H.; Hamm, B.; Wagner, S., Gadolinium-containing magnetic resonance contrast media: investigation on the possible transchelation of $\mathrm{Gd}(3)(+)$ to the glycosaminoglycan heparin. Contrast Media Mol. Imaging 2013, 8 (2), 108-16.

[48]. Frenzel, T.; Apte, C.; Jost, G.; Schockel, L.; Lohrke, J.; Pietsch, H., Quantification and Assessment of the Chemical Form of Residual Gadolinium in the Brain After Repeated Administration of Gadolinium-Based Contrast Agents: Comparative Study in Rats. Invest. Radiol. 2017, 52 (7), 396-404. 